\title{
Solution of the Skyrme-Hartree-Fock equations in the Cartesian deformed harmonic-oscillator basis. (III) HFODD (v1.75r): a new version of the program.
}

\author{
J.Dobaczewski ${ }^{a, b}$, and J. Dudek ${ }^{a, \text {, }}$ \\ ${ }^{a}$ Institute de Recherches Subatomiques, CNRS-IN ${ }_{2} P_{3} /$ Université Louis Pasteur, \\ F-67037 Strasbourg Cedex 2, France \\ ${ }^{b}$ Institute of Theoretical Physics, Warsaw University \\ ul. Hoża 69, PL-00681 Warsaw, Poland
}

\begin{abstract}
We describe the new version (v1.75r) of the code HFODD which solves the nuclear Skyrme-Hartree-Fock problem by using the Cartesian deformed harmonic-oscillator basis. Three minor errors that went undetected in the previous version have been corrected. The new version contains an interface to the LAPACK subroutine ZHPEV. Several methods of terminating the Hartree-Fock iteration procedure, and an algorithm that allows to follow the diabatic configurations, have been implemented.
\end{abstract}

PACS numbers: 07.05.T, 21.60.-n, 21.60.Jz

\section{NEW VERSION PROGRAM SUMMARY}

Title of the program: HFODD (v1.75r)

Catalogue number:

Program obtainable from: CPC Program Library, Queen's University of Belfast, N. Ireland (see application form in this issue)

Reference in CPC for earlier version of program: J. Dobaczewski and J. Dudek, Comput. Phys. Commun. 102 (1997) 183 (v1.60r).

Catalogue number of previous version: ADFL

Licensing provisions: none

Does the new version supersede the previous one: yes

Computers on which the program has been tested: CRAY C-90, SG Power Challenge L, IBM RS/6000, Pentium-II, Athlon

Operating systems: UNIX, UNICOS, IRIX, AIX, LINUX

\footnotetext{
${ }^{1}$ E-mail: jacek.dobaczewski@fuw.edu.pl

${ }^{2}$ E-mail: jerzy.dudek@ires.in2p3.fr
} 
Programming language used: FORTRAN-77

Memory required to execute with typical data: 10 Mwords

No. of bits in a word: The code is written in single-precision for the use on a 64-bit processor. The compiler option -r8 or +autodblpad (or equivalent) has to be used to promote all real and complex single-precision floating-point items to double precision when the code is used on a 32-bit machine.

Has the code been vectorised?: Yes

No. of lines in distributed program: 23987 (of which 10445 are comments and separators)

Keywords: Hartree-Fock; Skyrme interaction; Self-consistent mean-field; Nuclear manybody problem; Superdeformation; Quadrupole deformation; Octupole deformation; Pairing; Nuclear radii; Single-particle spectra; Nuclear rotation; High-spin states; Moments of inertia; Level crossings; Harmonic oscillator; Coulomb field; Point symmetries

Nature of physical problem

The nuclear mean-field and an analysis of its symmetries in realistic cases are the main ingredients of a description of nuclear states. Within the Local Density Approximation, or for a zero-range velocity-dependent Skyrme interaction, the nuclear mean-field is local and velocity dependent. The locality allows for an effective and fast solution of the self-consistent Hartree-Fock equations, even for heavy nuclei, and for various nucleonic ( $n$-particle $n$-hole) configurations, deformations, excitation energies, or angular momenta.

\section{Method of solution}

The program uses the Cartesian harmonic oscillator basis to expand single-particle wave functions of neutrons and protons interacting by means of the Skyrme effective interaction. The expansion coefficients are determined by the iterative diagonalization of the mean field Hamiltonians or Routhians which depend nonlinearly on the local neutron and proton densities. Suitable constraints are used to obtain states corresponding to a given configuration, deformation or angular momentum. The method of solution has been presented in: J. Dobaczewski and J. Dudek, Comput. Phys. Commun. 102 (1997) 166.

\section{Summary of revisions}

1. An error in the calculation of one of the time-odd mean-field potentials has been corrected.

2. A factor in the calculation of the multipole moment $Q_{22}$ has been corrected.

3. Scaling of the coupling constants has been corrected.

4. An interface to the LAPACK subroutine ZHPEV has been created.

5. Several methods of terminating the Hartree-Fock iteration procedure have been implemented.

6. An algorithm that allows to follow the diabatic configurations has been implemented.

7. Saving of auxiliary data for a faster calculation of the Coulomb potential has been implemented. 
8. Calculation of average quadrupole moments and radii of single-particle states has been added.

9. Calculation of the Bohr deformation parameters has been added.

Restrictions on the complexity of the problem

The main restriction is the CPU time required for calculations of heavy deformed nuclei and for a given precision required. One symmetry plane is assumed. Pairing correlations are only included in the BCS limit and for the conserved time-reversal symmetry (i.e., for non-rotating states in even-even nuclei).

Typical running time

One Hartree-Fock iteration for the superdeformed, rotating, parity conserving state of

${ }_{66}^{152} \mathrm{Dy}_{86}$ takes about nine seconds on the CRAY C-90 computer. Starting from the WoodsSaxon wave functions, about fifty iterations are required to obtain the energy converged within the precision of about $0.1 \mathrm{keV}$. In case when every value of the angular velocity is converged separately, the complete superdeformed band with precisely determined dynamical moments $\mathcal{J}^{(2)}$ can be obtained within one hour of CPU on the CRAY C-90, or within three hours of CPU on the Athlon-550 MHz processor. This time can be often reduced by a factor of three when a self-consistent solution for a given rotational frequency is used as a starting point for a neighboring rotational frequency.

Unusual features of the program

The user must have an access to the NAGLIB subroutine F02AXE or to the ESSL or LAPACK subroutine ZHPEV which diagonalize complex hermitian matrices, or provide another subroutine which can perform such a task. The LAPACK subroutine ZHPEV can be obtained from the Netlib Repository at University of Tennessee, Knoxville: http://netlib2.cs.utk.edu/cgi-bin/netlibfiles.pl?filename=/lapack/complex16/zhpev.t

\section{LONG WRITE-UP}

\section{Introduction}

The method of solving the Hartree-Fock (HF) equations in the Cartesian harmonic oscillator (HO) basis was described in the previous publication, Ref. [1], which is below referred to as I. The previous version of the code HFODD (v1.60r) was published in Ref. [2] which is below referred to as II. The present paper is a long write-up of the new version (v1.75r) of the code HFODD. This extended version is fully compatible with all previous versions.

Information provided in I and II remains valid, unless explicitly mentioned in the present long write-up.

In Section 2 we briefly review the modifications introduced in version (v1.75r) of the code HFODD. In particular, in Section 2.6 we present numerical tests of the code in situations when the iteration procedure does not converge because of a crossing of singleparticle levels in function of the rotational frequency. We then introduce a procedure to follow configurations along the so-called diabatic path. Such a procedure was implemented 
in version (v1.75r), and allows, in many cases, to obtain converged solutions for both crossing configurations.

Section 3 lists all additional new input keywords and data values, introduced in version (v1.75r). The structure of the input data file remains the same as in the previous versions, see Section 3 of II. Similarly, all previously introduced keywords and data values retain their validity and meaning.

\section{Modifications introduced in version (v1.75r)}

\subsection{Calculation of the density $\Delta s$}

There are two misprints in formulas (I-46) $]^{3}$ for the Cartesian components of the vector density $\Delta \boldsymbol{s}$. The correct expressions are as follows:

$$
\begin{array}{ll}
\Delta s_{1}= & 2 \Re\left(L^{+-}+L^{-+}\right)+2 T_{1}, \\
\Delta s_{2}= & -2 \Im\left(L^{+-}-L^{-+}\right)+2 T_{2}, \\
\Delta s_{3}= & 2 \Re\left(L^{++}-L^{--}\right)+2 T_{3} .
\end{array}
$$

The misprints were present only in the text of I, and did not affect the code HFODD (v1.60r). Unfortunately, the factors of 2, which should multiply densities $T_{1}$ and $T_{2}$ in Eqs. (1a) and (1b), respectively, where missing in version (v1.60r) of the code. As far as the numerical values are concerned, this error has been fairly unimportant for the final results, however, it may have created a weak spurious dependence of the results on the orientation of the nucleus with respect to the Cartesian reference frame, because only the $x$ and $y$ components of $\Delta s$ were affected.

Incorrect expressions for the densities $\Delta s_{1}$ and $\Delta s_{2}$ amounted to adding the erroneous term $-C_{t}^{\Delta s}\left(s_{x t} T_{x t}+s_{y t} T_{y t}\right)$ to the time-odd energy density $\mathcal{H}_{t}^{\text {odd }}(\boldsymbol{r})$ of Eq. (I-12a), and simultaneously adding the erroneous terms $-2 C_{t}^{\Delta s} T_{x t}$ and $-2 C_{t}^{\Delta s} T_{y t}$ to the time-odd spin potentials $\Sigma_{x t}$ and $\Sigma_{y t}$, respectively, Eq. (I-29b). Therefore, up to a very small difference between the matrix elements of spin and kinetic-spin potentials, the incorrect densities $\Delta s_{1}$ and $\Delta s_{2}$ where equivalent to a (direction-dependent) modification of the coupling constant $C_{t}^{T}$. This is the main reason why the error went undetected for a relatively long time. Needless to say, calculations performed with $C_{t}^{\Delta s}=0$, and in particular those with all time-odd terms neglected, are unaffected.

Since the term $\boldsymbol{s}_{t} \cdot \Delta \boldsymbol{s}_{t}$ gives anyhow fairly small contribution to the rotational properties of nuclei (compare curves denoted by open circles with those denoted by full squares in Figs. 3 and 4 of Ref. [3]), the incorrect expressions for densities $\Delta s_{1}$ and $\Delta s_{2}$ had numerically relatively small importance. The total energies could have been affected at the level of about $0.3 \mathrm{MeV}$ and the total spins at the level of about $0.3 \hbar$ (for details compare the output file reproduced in the section TEST RUN OUTPUT below with that given in II).

\footnotetext{
${ }^{3}$ Symbol (I-46) refers to Eq. (46) of I.
} 


\subsection{Calculation of the multipole moment $Q_{22}$}

In version (v1.60r) of the code HFODD, a factor of $\sqrt{6}$ was missing in the values of the multipole moment $Q_{22}$ printed on the output file.

Values of the quadrupole moments $Q_{20}$ and $Q_{22}$ depend on the normalization factors which in the standard nuclear-physics applications are different than those used in electrodynamics, cf. Ref. [4]. In particular, the code HFODD (v1.75r) uses the normalization factors such that

$$
\begin{aligned}
Q_{20} & =\left\langle 2 z^{2}-x^{2}-y^{2}\right\rangle \\
Q_{22} & =Q_{20} \times \tan \gamma
\end{aligned}
$$

where $\gamma$ is the standard angle measuring the non-axial quadrupole deformation [5].

\subsection{Scaling of the coupling constants}

As described in Section 3.2 of II, the code HFODD allows for the scaling of the Skyrmefunctional coupling constants by arbitrary factors which are read from the input data file. The scaling can be independently performed in the total-sum and isoscalar-isovector representations of the coupling constants, see Eqs. (I-14) and (I-15).

In version (v1.60r), the scaling formulas were incorrectly coded and as a result, under some very special circumstances described below, calculations were performed for values of the scaled coupling constants which were different from those intended by the user. This was happening provided that for the given coupling constant two conditions occurred simultaneously:

1. Scaling factor of the isovector coupling constant was different than 1 .

2. Scaling factor of the isovector coupling constant was different than that of the isoscalar coupling constant.

This is a fairly unusual combination of scaling conditions, and hence the error escaped detection for a relatively long time. In particular, all results obtained in Ref. [3] are correct because they were obtained with equal scaling factors of the isovector and isoscalar coupling constant, and hence condition 2. above was not fulfilled. Obviously, calculations performed without scaling, i.e., with all scaling factors equal 1, are unaffected.

\subsection{Diagonalization subroutines}

As described in Section 5.1, an interface to the LAPACK subroutine ZHPEV has been created. This allows using public-domain diagonalization subroutines, as an alternative of using the NAGLIB or ESSL packages, previously described in Section 5.3 of II.

\subsection{Terminating the Hartree-Fock iteration procedure}

As described in Section 3.1, the code HFODD (v1.75r) can detect several conditions which allow to stop the iteration procedure before the predefined number of iterations is exhausted. This includes stopping after the convergence is reached, and a required stability 
of the solutions is obtained, as well as an early stopping of the iteration procedure which is not going to converge.

\subsection{Diabatic blocking}

Crossing or non-crossing ("repulsion") of the mean-field single-particle energy levels in function of some continuous parameter(s) of theory, such as the rotational frequency and/or the constraining multipole moments, is one of the most important phenomena in the studies of high-spin nuclear states. They may give rise to several kinds of characteristic irregularities occurring along the rotational bands and these irregularities often help significantly in assigning the theoretical single-particle configurations to the experimental rotational bands.

As it is well known, levels that differ in terms of their discrete good quantum numbers such as, for instance, simplex, parity, signature ... etc., will generally cross. These crossings are such that the underlying intrinsic characteristics (e.g. single-particle alignments or multipole moments) do not change in any remarkable way before and after the crossing and this mechanism will be of no interest here.

In contrast, levels that belong to the same discrete symmetry will most of the time approach each other in function of the parameter studied and then go apart but in such a way that the intrinsic characteristics of the first one will go over to the second one and vice versa. This non-crossing rule, sometimes called the Landau-Zener effect, cannot be given any more rigorous general formulation, and it may happen that the distance of the closest approach for the same symmetry levels is zero. In those, in practice very rare cases, the two crossing mechanisms mentioned do not differ.

An example of a crossing of two HF configurations (no pairing) is presented in Fig. 1. The left panel shows the total energies $E(I)$ as functions of the total spin $I$, while the right panel shows the total Routhians $R(\omega)=E(\omega)-I(\omega) \omega$ as functions of the rotational frequency $\hbar \omega$. Since both quantities vary very rapidly as functions of their arguments, they are plotted with respect to the corresponding rigid-rotor reference values, i.e., $E(I)$ is shown relatively to $I(I+1) /\left(2 J_{0}\right)$, and $R(\omega)$ is shown relatively to $-J_{0} \omega^{2} / 2$, where the constant rigid-rotor moment of inertia of $J_{0}=100 \hbar^{2} / \mathrm{MeV}$ has been used. Calculations have been performed within the cranking approximation for values of the rotational frequency of $\hbar \omega=0.5(0.05) 0.8 \mathrm{MeV}$.

The examples shown in Fig. 1 correspond to two bands in ${ }^{151} \mathrm{~Tb}$ (see Ref. [6] for a more complete description of calculations performed in this nucleus). Both configurations contain the same set of the single-particle levels of ${ }^{150} \mathrm{~Tb}$ being occupied. They correspond to the neutron and proton configurations of $\left(N_{++}, N_{+-}, N_{-+}, N_{--}\right)=(22,21,21,21)$ and $(15,16,17,17)$, respectively, as described by the numbers of states occupied in the paritysignature blocks $(\pi, r)=(+1,+i),(+1,-i),(-1,+i)$, and $(-1,-i)$. The ground-state band in ${ }^{151} \mathrm{~Tb}$ can be obtained by putting the 86th neutron into the lowest available level for $N_{+-}=22$, thus obtaining the closed $N=86$ SD magic neutron configuration. Since the order of orbitals may change with changing rotational frequency, numbers $N_{\pi,-i r}$ are not necessarily the most practical for defining physical characteristics of the single-particle states in question. Usually, one uses the so-called asymptotic Nilsson quantum numbers $\left[N n_{z} \Lambda\right] \Omega$ [5] for that purpose. Code HFODD calculates these quantum numbers by finding 
the dominant Nilsson components of the HF single-particle states. Several excited bands in ${ }^{151} \mathrm{~Tb}$ can be obtained by putting the 86th neutron into one of the higher available levels, for instance, in the $N_{-+}=22,23$, or 24 levels, that correspond to one of the [521]3/2(r=+i), $[514] 9 / 2(r=+i)$, or $[761] 3 / 2(r=+i)$ Nilsson orbitals.

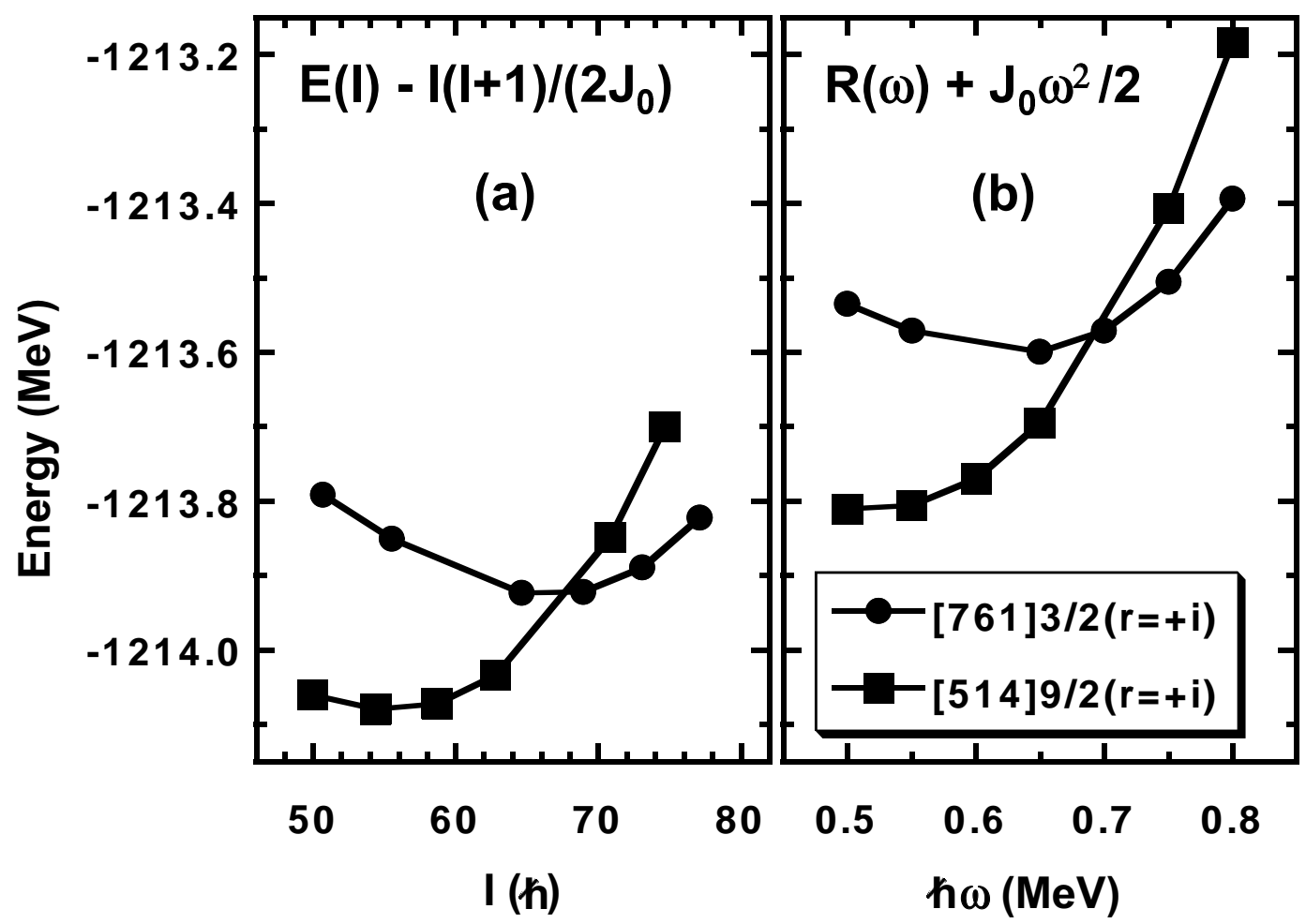

Figure 1: Total energies $E(I),(a)$, and total Routhians $R(\omega)$, (b), of the [761]3/2(r=+i) and [514] $9 / 2(r=+i)$ neutron diabatic configurations in ${ }^{151} \mathrm{~Tb}$. The rigid-rotor reference energies for $J_{0}=100 \hbar^{2} / \mathrm{MeV}$ have been subtracted. (In our graphical representation we employ the convention according to which the levels that carry the smoothly varying intrinsic characteristics (see text) are denoted with the same symbols. This is at variance with the convention that stresses the Landau-Zener mechanism, as used by some authors. According to the latter one, the sequences of the lowest-lying points would have been drawn as squares and that of the higher-lying points as circles.)

Of particular interest in ${ }^{151} \mathrm{~Tb}$ are exited bands in which the [761]3/2(r=+i) intruder orbital is occupied. This orbital decreases in energy with increasing rotational frequency, and therefore, it crosses the other $(\pi, r)=(-1,+i)$ orbitals. In particular, in ${ }^{151} \mathrm{~Tb}$ the [761]3/2 $(r=+i)$ orbital corresponds to the $N_{-+}=24$ th orbital at low frequencies, then it becomes the $N_{-+}=23 \mathrm{rd}$ orbital, and finally, at high frequencies it is the lowest available $N_{-+}=22$ nd orbital. Configurations shown in Fig. 1 correspond to the crossing of the [761]3/2 $(r=+i)$ and [514]9/2(r=+i) orbitals. Following the standard convention, these configurations are called the diabatic ones, because they correspond to the given orbital being occupied, irrespective of its excitation energy. On the other hand, configurations 
based on occupying the $N_{-+}=22,23$, or 24 states are called the adiabatic ones. Obviously, in adiabatic configurations, different Nilsson orbitals are occupied at different frequencies.

Fig. 2 shows the negative-parity single-particle neutron Routhians in ${ }^{151} \mathrm{~Tb}$. The left and right panels show the results obtained for the $[761] 3 / 2(r=+i)$ and $[514] 9 / 2(r=+i)$ diabatic configurations, respectively. The occupied orbitals are in both cases denoted by the filled symbols. It is clear that the crossing frequency depends on which of the shown orbitals is occupied. This is due to the self-consistent effects that influence the deformations, spins, and other characteristics of many-body states, calculated at given rotational frequencies and for given particle-hole configurations.

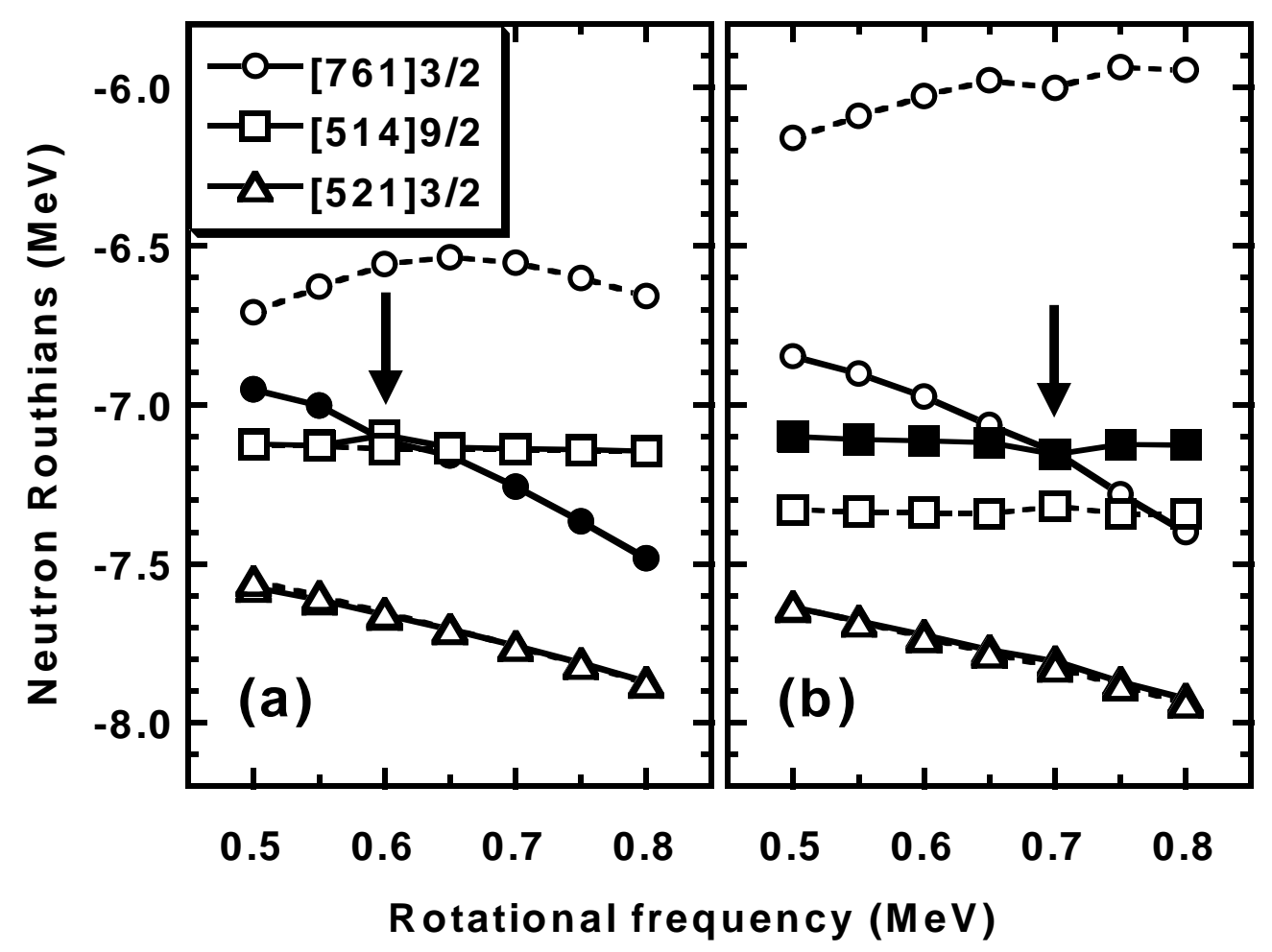

Figure 2: Negative-parity neutron single-particle Routhians in ${ }^{151} \mathrm{~Tb}$ calculated for the [761]3/2( $r=+i)(\mathrm{a})$ and [514] $9 / 2(r=+i)$ (b) neutron diabatic configurations. Solid and dashed curves denote the $r=+i$ and $r=-i$ signatures, respectively. The arrows denote the angular frequencies where the converged solutions near the crossing points could not be found.

In the calculations, one has to begin the analysis by finding the adiabatic configurations, in order to find the lowest available orbitals, to determine their physical characteristics, and to check whether or not there are any crossings of configurations that should be followed diabatically. The input data files tb151-a.dat and tb151-b.dat give examples of runs that find the adiabatic configurations for the $N_{-+}=23 \mathrm{rd}$ and $N_{-+}=24$ th orbitals 
being occupied, respectively.

After several HF iterations, the levels of the same symmetry, that are energetically sufficiently far, change their intrinsic characteristics (such as, e.g., angular momentum alignments) only by very small amounts. This is not true for the case of the crossing when the two states in question exchange their relative positions from one iteration to another. Therefore, in the HF calculations, very often the Landau-Zener avoided crossings of levels manifest themselves in the form of diverging iteration procedure. This observation is used by the code HFODD (v1.75r) in order to locate the crossing by recognizing the oscillatory ("ping-pong") behavior of solutions.

Table 1: Example of the output printed when the "ping-pong" divergence is found, see text.

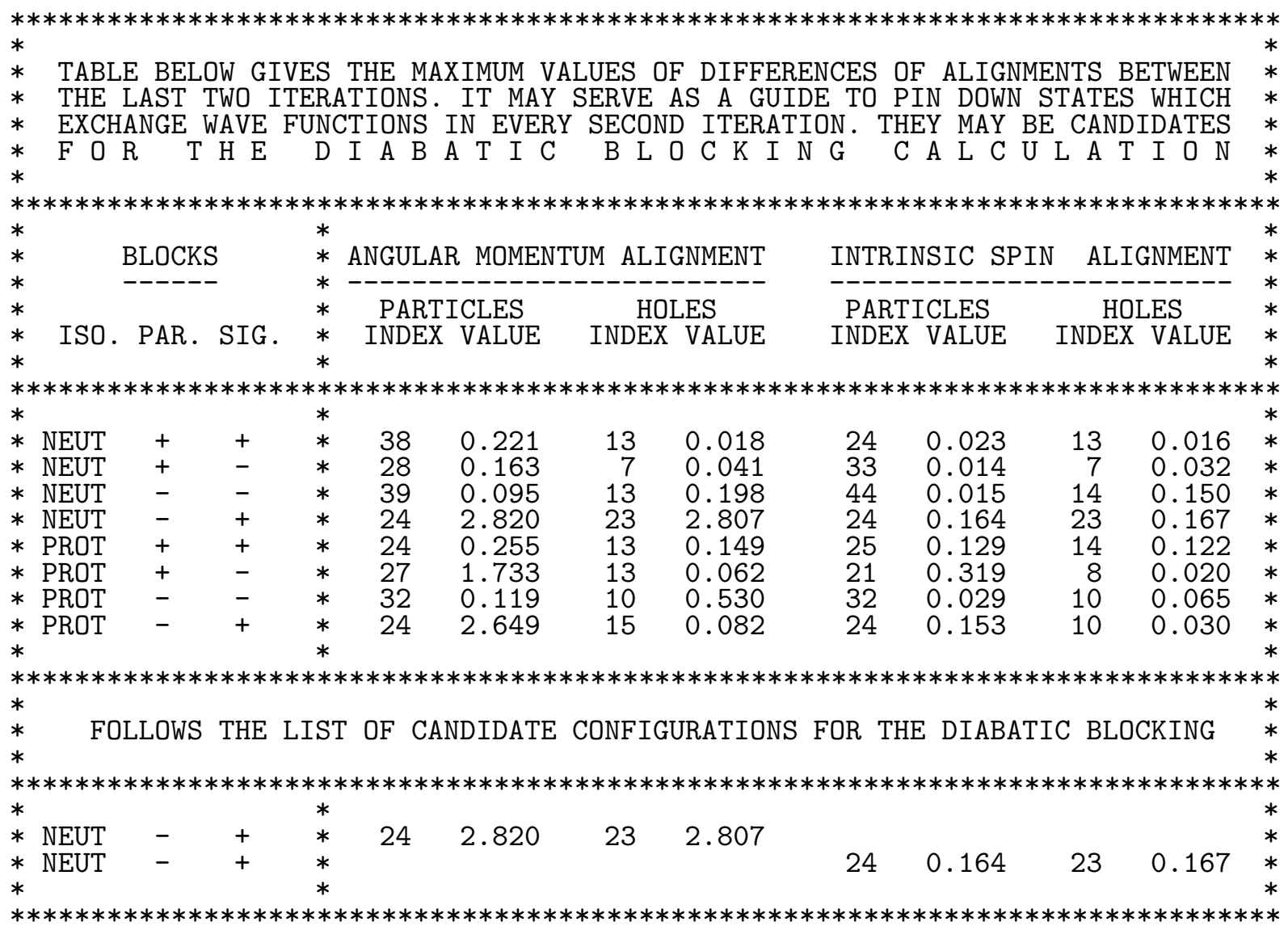

The "ping-pong" divergence is a result of the standard self-consistent prescription, for which the occupied states in a given iteration are the eigenstates of the mean-field Hamiltonian diagonalized in the preceding iteration. Such a divergence is characterized by two series of HF states, each one appearing in every second HF iteration. Focusing our attention on the specific example discussed above, one can describe such a divergence in the following way. Suppose that in a given HF iteration, the single-particle orbital $[761] 3 / 2(r=+i)$ appears below the $[514] 9 / 2(r=+i)$ orbital, i.e., it corresponds to 
$N_{-+}=23$. In this particular iteration it becomes occupied, and is included in all densities, for which the mean-field Hamiltonian is determined. In the next iteration, the new Hamiltonian is diagonalized, however, at present the orbital [514]9/2( $r=+i)$ appears below $[761] 3 / 2(r=+i)$, and it is this orbital, again for $N_{-+}=23$, which becomes occupied. It is obvious that such a situation may repeat itself infinitely many times, never leading to a self-consistent solution.

In many cases, it is possible to find converged solutions near the crossing points simply by decreasing the convergence rate, i.e., by using parameters SLOWEV and SLOWOD (see Sec. 3.5 of II) only slightly smaller then 1 . This method works well provided that the two crossing orbitals have fairly different characteristics, like it is the case in the example discussed here. However, in the case of a strong mixing between the two crossing orbitals, the HF iteration procedure converges near the crossing points only seldomly.

Upon recognizing the "ping-pong" divergence condition (see Sec. 3.1 for details of the procedure), the code HFODD (v1.75r) prints the summary table that may help in identifying the crossing orbitals. For the $N_{-+}=23$ adiabatic configuration at $\hbar \omega=0.65 \mathrm{MeV}$, the table has the form presented in Table 1 .

In the upper part of the table, the code prints the absolute values of differences $\left|\Delta i_{y}\right|$ $\left(\left|\Delta s_{y}\right|\right)$ of single-particle average alignments $i_{y}$ (intrinsic spins $s_{y}$ ) between the last and the last but one iterations (columns denoted by VALUE). The results are printed separately for the particle (empty) and hole (occupied) states, and their indices are given in columns denoted by INDEX. In each of the charge-parity-signature (or charge-simplex) blocks the results are printed only for those states for which the values of $\left|\Delta i_{y}\right|$ and $\left|\Delta s_{y}\right|$ are the largest ones.

In the lower part of the table, the code prints the list of particle-hole pairs from the upper part of the table, for which the indices (INDEX) differ by one, i.e., which correspond to the particle-hole pairs at the Fermi surface. In the specific example discussed here, the code properly identifies the crossing of the $N_{-+}=23$ and $N_{-+}=24$ orbitals, and proposes the diabatic blocking based on the average values of the single-particle alignments, see Sec. 3.2. The crossing orbitals, [761]3/2(r=+i) and [514]9/2(r=+i), have markedly different alignments of about $i_{y}=+2.23$ and $-0.46 \hbar$, respectively. This corresponds to values of $\left|\Delta i_{y}\right|=+2.820$ or $2.807 \hbar$ printed in the table. Therefore, by requesting that the state that has a larger alignment be occupied (among the $N_{-+}=23$ and $N_{-+}=24$ orbitals), one obtains the diabatic [761]3/2( $r=+i)$ configuration. Similarly, by choosing the state that has a smaller alignment, one obtains the [514] $9 / 2(r=+i)$ diabatic configuration, and this is so irrespective of which orbital has smaller or larger energy, i.e., irrespective of the angular frequency. The input data files tb151-c.dat and tb151-d.dat give examples of runs that find the diabatic configurations for the $[761] 3 / 2(r=+i)$ and $[514] 9 / 2(r=+i)$ orbitals being occupied, respectively.

\subsection{Saving the Coulomb data}

As described in Section 3.3, the code HFODD (v1.75r) can save on disk auxiliary data for a faster calculation of the Coulomb potential. These data can be reused in subsequent runs of the code, provided the Coulomb parameters NUMCOU, NUMETA, and FURMAX (see Section 3.5 of II) do not change from one run to the next. 


\subsection{Calculation of moments and radii}

Average quadrupole moments, $\left\langle q_{20}\right\rangle$ and $\left\langle q_{22}\right\rangle$, radii squared, $\left\langle r^{2}\right\rangle$, and sizes squared, $\left\langle x^{2}\right\rangle$, $\left\langle y^{2}\right\rangle$, and $\left\langle z^{2}\right\rangle$, of single-particle states are calculated and stored in the review file FILREV, see Section 3.9 of II.

\subsection{Calculation of the Bohr deformation parameters}

In version (v1.75r), the Bohr deformation parameters $\alpha_{\lambda \mu}$ [5] are calculated using the firstorder approximation. The code uses the simplest linearized expression (cf. Eq. (1.35) of Ref. [5]) relating deformation parameters to multipole moments of a sharp-edge uniformly charged shape, i.e.,

$$
\alpha_{\lambda \mu}=\frac{4 \pi \sqrt{1+\delta_{\mu 0}}}{3 N R_{0}^{\lambda}}\left\langle r^{\lambda} Y_{\lambda \mu}\right\rangle .
$$

For $\mu \neq 0$ the deformation parameters contain the standard factor of $\sqrt{2}$. This reflects the fact that for the assumed $\hat{S}_{y}$ simplex symmetry (see Sec. 3.1 of I), the values of multipole moments for $\mu<0$ are up to a phase equal to those for $\mu>0$. Neutron, proton, or mass deformation parameters are calculated from the corresponding neutron, proton, or mass multipole moments $\left\langle r^{\lambda} Y_{\lambda \mu}\right\rangle$, and by setting $N$ in Eq. (3) equal to the number of neutrons, protons, or nucleons. The equivalent radii $R_{0}$ are respectively calculated from the neutron,

proton, or mass rms radii as $\sqrt{5 / 3}\left\langle r^{2}\right\rangle^{1 / 2}$. One should note that for large deformations the neglected higher-order terms (see Eq. (1.35) of Ref. [5]) will in general be non-negligible. Therefore, the printed values of the Bohr deformation parameters should be used only as (often very) rough estimates.

\section{Input data file}

Structure of the input data file has been described in II, and in version (v1.75r) of the code HFODD this structure is exactly the same. All previous items of the input data file remain valid, and several new items were added, as described in Secs. 3.1 3.3.

Together with the FORTRAN source code in the file hfodd.f, several examples of the input data files are provided. File dy152-c.dat contains all the valid input items, and the input data are identical to the default values. Therefore, the results of running the code with the input data file dy152-c.dat are identical to those obtained for the input data file containing only one line with the keyword EXECUTE.

File dy152-d. dat contains all those input items that were added in version (v1.75r), and the input data there are those recommended for being used in the new version. This file is reproduced in the section TEST RUN INPUT below.

\subsection{General data}

Keyword: ITERAT_EPS

$$
0.0=\text { EPSITE }
$$

Keyword: MAXANTIOSC

$$
1=\text { NULAST }
$$


These two parameters govern the termination of the HF iterations according to the achieved stability of solutions. The stability of the HF energy has been defined in (I-37) as the difference between the total energies calculated from the single-particle energies and from the Skyrme functional. The HF iterations continue until the absolute value of the stability is smaller than EPSITE (in MeV) over NULAST consecutive iterations. When this condition is fulfilled, iteration procedure terminates and the final results are printed. This allows for an automated adjustment of the number of iterations which are required to achieve a given level of convergence. The number of iterations NOITER, Section 3.1 of II, can now be set to a large value at which the iterations terminate if a stable solution is not found.

The default value of EPSITE $=0.0$ ensures that whenever this new option is not used, the code HFODD (v1.75r) behaves as that in version (v1.60r). If a non-zero value of EPSITE is used, a non-zero value of NULAST has to be used too. In practice, a value of NULAST $=5$ prevents an accidental termination of iterations in all cases when the stability energy changes the sign, but the solution is not yet self-consistent.

Keyword: PING-PONG

$$
0.0,3=\text { EPSPNG, NUPING }
$$

The code is able to detect the "ping-pong" divergence described in Section 2.6, i.e., the situation when the HF iteration procedure gives alternating results in every second iteration. Upon continuing the iteration, both sequences of results, i.e., those which correspond to the iteration numbers being even and odd, stay different but perfectly stable, and hence the correct self-consistent solution is never attained.

The code recognizes such a situation by calculating the averages and variances of the stability energy (I-37), separately in the even and in the odd sequences of results, over the last NUPING pairs of iterations. The "ping-pong" divergence condition occurs when both variances become a factor EPSPNG smaller than the absolute value of the difference of the corresponding averages, i.e., when

$$
\begin{aligned}
\Delta(\delta \mathcal{E})_{\text {even }} & <\text { EPSPNG } \times\left|\overline{\delta \mathcal{E}}_{\text {even }}-\overline{\delta \mathcal{E}}_{\text {odd }}\right| \\
\Delta(\delta \mathcal{E})_{\text {odd }} & <\operatorname{EPSPNG} \times\left|\overline{\delta \mathcal{E}}_{\text {even }}-\overline{\delta \mathcal{E}}_{\text {odd }}\right|
\end{aligned}
$$

where

$$
\begin{aligned}
\overline{\delta \mathcal{E}}_{\text {even }} & =\left(\sum_{p=0}^{\mathrm{NUPING}-1} \delta \mathcal{E}_{n-2 p}\right) / \text { NUPING } \\
\overline{\delta \mathcal{E}}_{\text {odd }} & =\left(\sum_{p=0}^{\mathrm{NUPING}-1} \delta \mathcal{E}_{n-2 p-1}\right) / \text { NUPING }
\end{aligned}
$$

and

$$
\begin{aligned}
\Delta(\delta \mathcal{E})_{\text {even }} & =\left(\sum_{p=0}^{\text {NUPING }-1}\left(\delta \mathcal{E}_{n-2 p}-\overline{\delta \mathcal{E}}_{\text {even }}\right)^{2}\right)^{1 / 2} / \text { NUPING } \\
\Delta(\delta \mathcal{E})_{\text {odd }} & =\left(\sum_{p=0}^{\text {NUPING }-1}\left(\delta \mathcal{E}_{n-2 p-1}-\overline{\delta \mathcal{E}}_{\text {odd }}\right)^{2}\right)^{1 / 2} / \text { NUPING. }
\end{aligned}
$$


Here, $n$ denotes the number of the last accomplished HF iteration, and $\delta \mathcal{E}_{n^{\prime}}$ denotes the stability energy (I-37) obtained in the $n^{\prime}$-th iteration.

The default value of $E P S P N G=0.0$ ensures that whenever this new option is not used, the code HFODD (v1.75r) behaves as that in version (v1.60r). If a non-zero value of EPSPNG is used, a value of NUPING $>1$ has to be used too. In practice, values of EPSPNG $=0.01$ and $N U P I N G=3$ allow for an efficient detection of the "ping-pong" divergence condition.

Upon discovering the "ping-pong" divergence, the HF iterations are terminated and a table of absolute values of maximum differences of single-particle observables between the two sequences of iterations is printed, see Sec. 2.6 and Table 1. These maximum differences are determined for states in each of the charge-parity-signature (or chargesimplex) blocks, and separately for particle and hole states. Whenever such a maximum difference is found for a particle state and for a hole state with adjacent indices, such a pair is proposed as a candidate for the diabatic blocking calculation, see Secs. 2.6 and 3.2 .

Keyword: CHAOTIC

$$
0=\text { NUCHAO }
$$

The code is able to detect the "chaotic" divergence which occurs when the HF iterations give results which chaotically vary from one iteration to another one. The code recognizes such a divergence by finding the local maxima $M_{k}, k=1,2, \ldots$, in the sequence of absolute values of the stability energies (I-37), obtained in the entire series of the HF iterations performed. The "chaotic" divergence condition occurs when the code finds NUCHAO positive differences $M_{k}-M_{k-1}$. When this condition occurs, iteration procedure terminates and the final results are printed.

For $\mathrm{NUCHAO}=0$ (the default value) the code does not check whether the "chaotic" divergence occurs or not. In practice, a value of $\mathrm{NUCHAO}=5$ allows for an efficient detection of the "chaotic" divergence condition. However, for a small value of NUCHAO and a small value of EPSPNG, the "ping-pong" divergence can sometimes be mistaken for the "chaotic" divergence. If one is interested in the diabatic-blocking data, printed after the "ping-pong" divergence, the recommended value of $\mathrm{NUCHAO}=5$ should be increased to 10 or more.

Keyword: PHASESPACE

$$
\begin{aligned}
0,0,0,0= & \operatorname{NUMBSP}(0,0), \operatorname{NUMBSP}(1,0), \\
& \operatorname{NumBSP}(0,1), \operatorname{NUMBSP}(1,1)
\end{aligned}
$$

Numbers of the lowest mean-field eigenstates which are kept after the diagonalization of the mean-field Hamiltonians in the four simplex-charge blocks: $(s, q)=(+i, n),(-i, n)$, $(+i, p),(-i, p)$. All other eigenstates are discarded. If any of these numbers is equal to zero (the default value), the code sets it equal to the number of neutrons IN_FIX (for $q=n$ ) or protons IZ_FIX (for $q=p$ ), see Section 3.1 of II.

For calculations without pairing, the user is responsible for using values of NUMBSP large enough to accommodate all wave functions which might be useful for the required vacuum and particle-hole configurations, see Section 3.4 of II. In practice, the use of the default values described above is recommended as a safe option. The execution time is almost independent of NUMBSP. The size of the matrices defined by the NDSTAT parameter can be reduced by the user for smaller values of NUMBSP. 


\subsection{Configurations}

Keyword: DIASIM_NEU

$$
\begin{aligned}
& 2,2, \quad 1,1, \quad 0,0=\operatorname{KPFLIM}(0,0), \operatorname{KPFLIM}(1,0), \\
& \operatorname{KHFLIM}(0,0), \operatorname{KHFLIM}(1,0) \text {, } \\
& \operatorname{KOFLIM}(0,0), \operatorname{KOFLIM}(1,0)
\end{aligned}
$$

Diabatic blocking of neutron single-particle simplex configurations. Matrices KPFLIM contain the indices of the particle states in the two simplex blocks denoted by $(+)$ and $(-)$, of given simplex values, i.e., $s=+i$ and $-i$, respectively. Matrices KHFLIM contain analogous indices of the hole states, and matrices KOFLIM define type of blocking according to the following table:

KOFLIM $=0 \Longleftrightarrow$ No diabatic blocking in the given parity/signature block.
KOFLIM $=+1 \Longleftrightarrow$ The state which has the larger alignment is occupied.
KOFLIM $=-1 \Longleftrightarrow$ The state which has the smaller alignment is occupied.
KOFLIM $=+2 \Longleftrightarrow$ The state which has the larger intrinsic spin is occupied.
KOFLIM=-2 $\Longleftrightarrow$ The state which has the smaller intrinsic spin is occupied.

Within the diabatic blocking procedure one does not predefine whether the particle or the hole state is occupied (like is the case when the particle-hole excitations are defined, see Section 3.4 of II). In each iteration the code calculates the average alignments (or average intrinsic spins) of both states (those defined by KPFLIM and KHFLIM), and occupies that state for which a larger, or a smaller value is obtained. Therefore, the order of both states in the Routhian spectrum is irrelevant.

The user is responsible for choosing the particle-state indices (in KPFLIM) only among those corresponding to empty single-particle states, and the hole-state indices (in KHFLIM) only among those corresponding to occupied single-particle states, see Section 3.4 of II.

Keyword: DIASIM_PRO

$$
\begin{aligned}
& 2,2, \quad 1,1, \quad 0,0=\operatorname{KpFLiM}(0,1), \operatorname{KPFLiM}(1,1), \\
& \operatorname{KHFLIM}(0,1), \operatorname{KHFLIM}(1,1) \text {, } \\
& \operatorname{KOFLIM}(0,1), \operatorname{KOFLIM}(1,1)
\end{aligned}
$$

Same as above but for the diabatic blocking of proton single-particle simplex configurations.

Keyword: DIASIG_NEU

$$
\begin{aligned}
& 2,2,2,2, \quad 1,1,1,1, \quad 0,0,0,0= \\
& \operatorname{KPFLIG}(0,0,0), \operatorname{KPFLIG}(0,1,0), \operatorname{KPFLiG}(1,0,0), \operatorname{KPFLIG}(1,1,0), \\
& \operatorname{KhFLiG}(0,0,0), \operatorname{KHFLiG}(0,1,0), \operatorname{KHFLiG}(1,0,0), \operatorname{KHFLIG}(1,1,0), \\
& \operatorname{KOFLIG}(0,0,0), \operatorname{KOFLIG}(0,1,0), \operatorname{KOFLIG}(1,0,0), \operatorname{KOFLIG}(1,1,0)
\end{aligned}
$$

Diabatic blocking of neutron single-particle parity/signature configurations. Matrices KPFLIG contain the indices of particle states in the four parity/signature blocks denoted by $(+,+),(+,-),(-,+)$, and $(-,-)$, of given (parity,signature) combinations, i.e., $(\pi, r)=(+1,+i),(+1,-i),(-1,+i)$, and $(-1,-i)$, respectively. Matrices KHFLIG contain analogous indices of hole states, and matrices KOFLIG define the type of blocking according to the table of values identical to that defined for the simplex case above. Other rules described for the simplex case apply here analogously. 
Keyword: DIASIG_PRO

$$
\begin{aligned}
& 2,2,2,2, \quad 1,1,1,1, \quad 0,0,0,0= \\
& \operatorname{KPFLIG}(0,0,1), \operatorname{KPFLIG}(0,1,1), \operatorname{KPFLIG}(1,0,1), \operatorname{KPFLIG}(1,1,1), \\
& \operatorname{KHFLIG}(0,0,1), \operatorname{KHFLIG}(0,1,1), \operatorname{KHFLIG}(1,0,1), \operatorname{KHFLIG}(1,1,1), \\
& \operatorname{KOFLIG}(0,0,1), \operatorname{KOFLIG}(0,1,1), \operatorname{KOFLIG}(1,0,1), \operatorname{KOFLIG}(1,1,1)
\end{aligned}
$$

Same as above but for the diabatic blocking of proton single-particle parity/signature configurations.

\section{$3.3 \quad$ Files}

Keyword: COULOMFILE

\section{HFODD.COU = FILCOU}

CHARACTER*68 file name of the file containing auxiliary data for a faster calculation of the Coulomb potential. Must start at the 13-th column of the data line. Parameters NUMCOU, NUMETA, and FURMAX which define the calculations of the direct Coulomb potential (see Section 5 of I and Section 3.5 of II) are usually kept unchanged for the whole series of calculations in one region of nuclei. Therefore, many Coulomb auxiliary results can be calculated only once, and stored in the file FILCOU. Handling of this file is determined by the input parameters ICOULI and ICOULO.

Keyword: COULOMSAVE

$$
0,0=\text { ICOULI, ICOULO }
$$

Input parameters ICOULI and ICOULO determine actions pertaining to reading and/or writing of the Coulomb file FILCOU, according to the following table:

\begin{tabular}{ccl} 
ICOULI & ICOULO & \multicolumn{1}{c}{ Action } \\
\hline 0 & 0 & neither read nor write the Coulomb file \\
1 & 0 & read, but do not write the Coulomb file \\
0 & 1 & do not read, but write the Coulomb file \\
1 & 1 & use automated handling of the Coulomb file \\
\hline
\end{tabular}

The default values of ICOULI $=I C O U L O=0$ ensure that whenever this new option is not used, the code HFODD (v1.75r) behaves as that in version (v1.60r). However, unless it is required by special circumstances, a use of the automated handling of the Coulomb file $(I C O U L I=I C O U L O=1)$ is recommended. Within the automated mode, the code checks whether the Coulomb file exists, and whether it contains data which match the current values of the input parameters NUMCOU, NUMETA, and FURMAX. If this is the case, the code reads the data from the Coulomb file. If this is not the case, the code calculates the Coulomb auxiliary results and stores them in the Coulomb file FILCOU. In the automated mode, the user is informed by appropriate messages printed on the output file about what type of the action has been taken in the given run of the code.

\section{Output file}

Together with the FORTRAN source code in the file hfodd.f, an example of the output file is provided in dy152-d.out. Selected lines from this file are presented in the section 
TEST RUN OUTPUT below. This output file corresponds to the input file dy152-d.dat reproduced in the section TEST RUN INPUT below.

\section{FORTRAN source file}

The FORTRAN source code in is provided in the file hfodd.f and can be modified in several places, as described in this section.

\subsection{Library subroutines}

The code HFODD requires an external subroutine which diagonalizes complex hermitian matrices. Version (v1.75r) contains an interface to the LAPACK subroutine ZHPEV that can be downloaded (with dependencies) from http://netlib2.cs.utk.edu/cgi-bin/netlibfiles.pl?filename=/lapack/complex16/zhpev. This subroutine and its dependencies are in the REAL*8 and COMPLEX*16 versions, and should be compiled without promoting real numbers to the double precision. On the other hand, the code HFODD itself does require compilation with an option promoting to double precision. Therefore, the code and the ZHPEV package should be compiled separately, and then should be linked together.

In order to activate the interface to the LAPACK ZHPEV subroutine, the following modifications of the code HFODD (v1.75r) have to be made:

1. Change everywhere the value of parameter I_CRAY=1 into I_CRAY=0.

2. Change everywhere the value of parameter IZHPEV=0 into IZHPEV=1.

3. Change the name of the subroutine ZHPEV, provided with the code HFODD, to another name, or remove it from the file.

4. If your compiler does not support undefined externals, or subroutines called with different parameters, remove calls to subroutines CGEMM, F02AXE, and the first call to ZHPEV.

\section{Acknowledgments}

Useful comments and tests of the code performed by M. Bender, H. Molique, W. Satuła, and T.R. Werner are gratefully acknowledged. This research was supported in part by the Polish Committee for Scientific Research (KBN) under Contract No. 2 P03B 040 14, by the French-Polish integrated actions programme POLONIUM, and by the computational grant from the Interdisciplinary Centre for Mathematical and Computational Modeling (ICM) of the Warsaw University.

\section{References}

[1] J. Dobaczewski and J. Dudek, Comput. Phys. Commun. 102, 166 (1997).

[2] J. Dobaczewski and J. Dudek, Comput. Phys. Commun. 102, 183 (1997). 
[3] J. Dobaczewski and J. Dudek, Phys. Rev. C52, 1827 (1995).

[4] J.D. Jackson, Classical Electrodynamics (Wiley, New York, 1975).

[5] P. Ring and P. Schuck, The Nuclear Many-Body Problem (Springer-Verlag, Berlin, 1980).

[6] N. El Aouad, J.Dobaczewski, J. Dudek, X. Li, W.D. Luo, H. Molique, A. Bouguettoucha, Th. Byrski, F.A. Beck, D. Curien, G. Duchêne, Ch. Finck, and B. Kharraja, submitted to Nuclear Physics A. 


\section{TEST RUN INPUT}

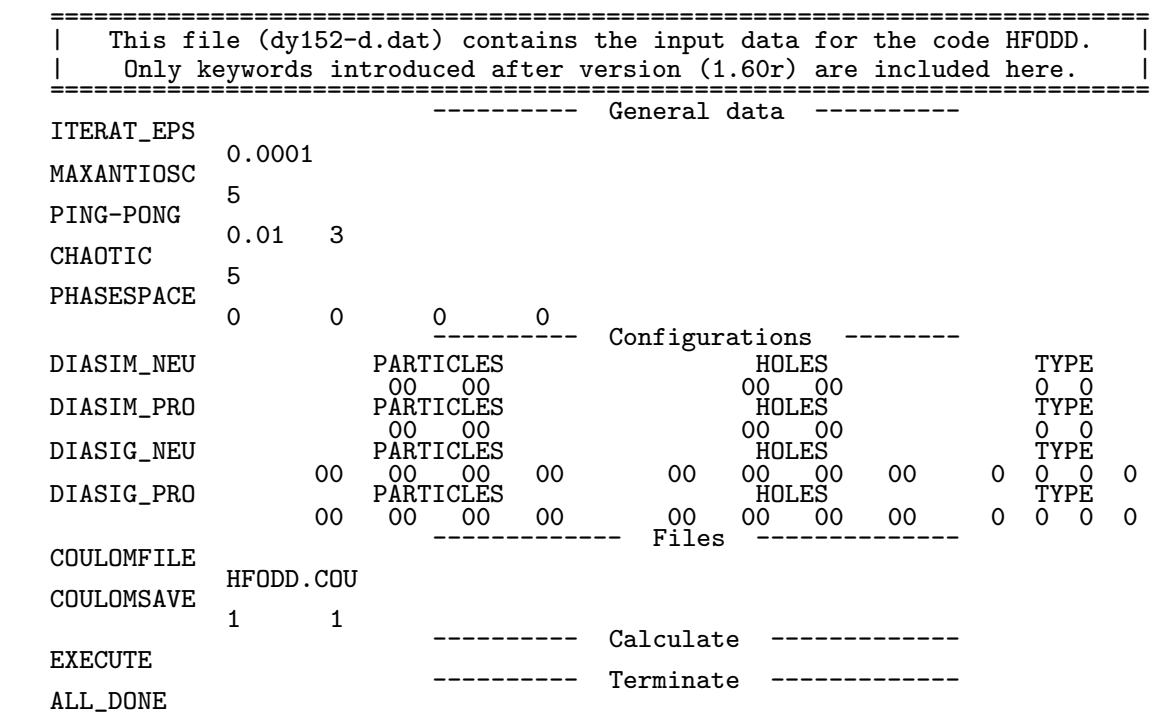




\section{TEST RUN OUTPUT}

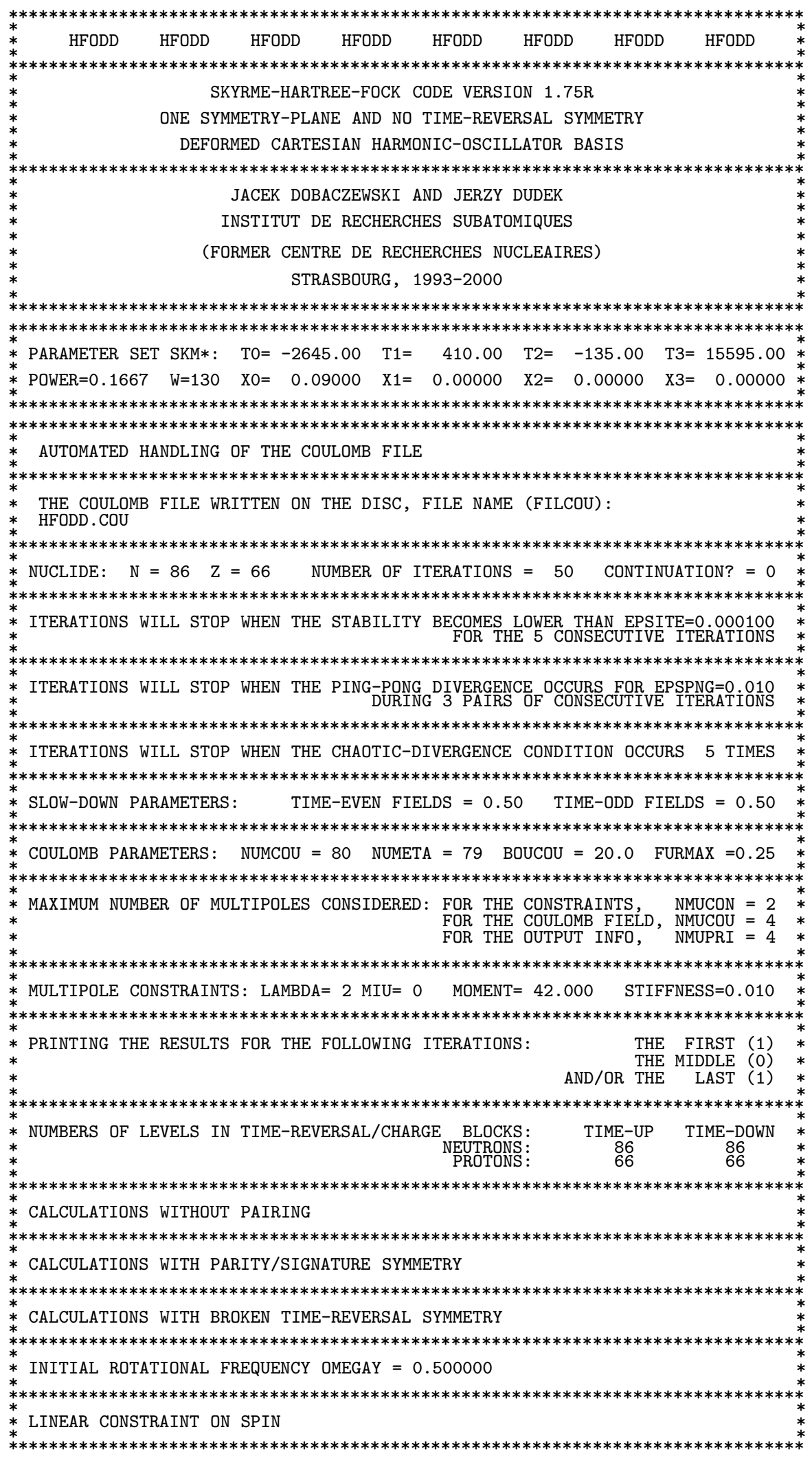




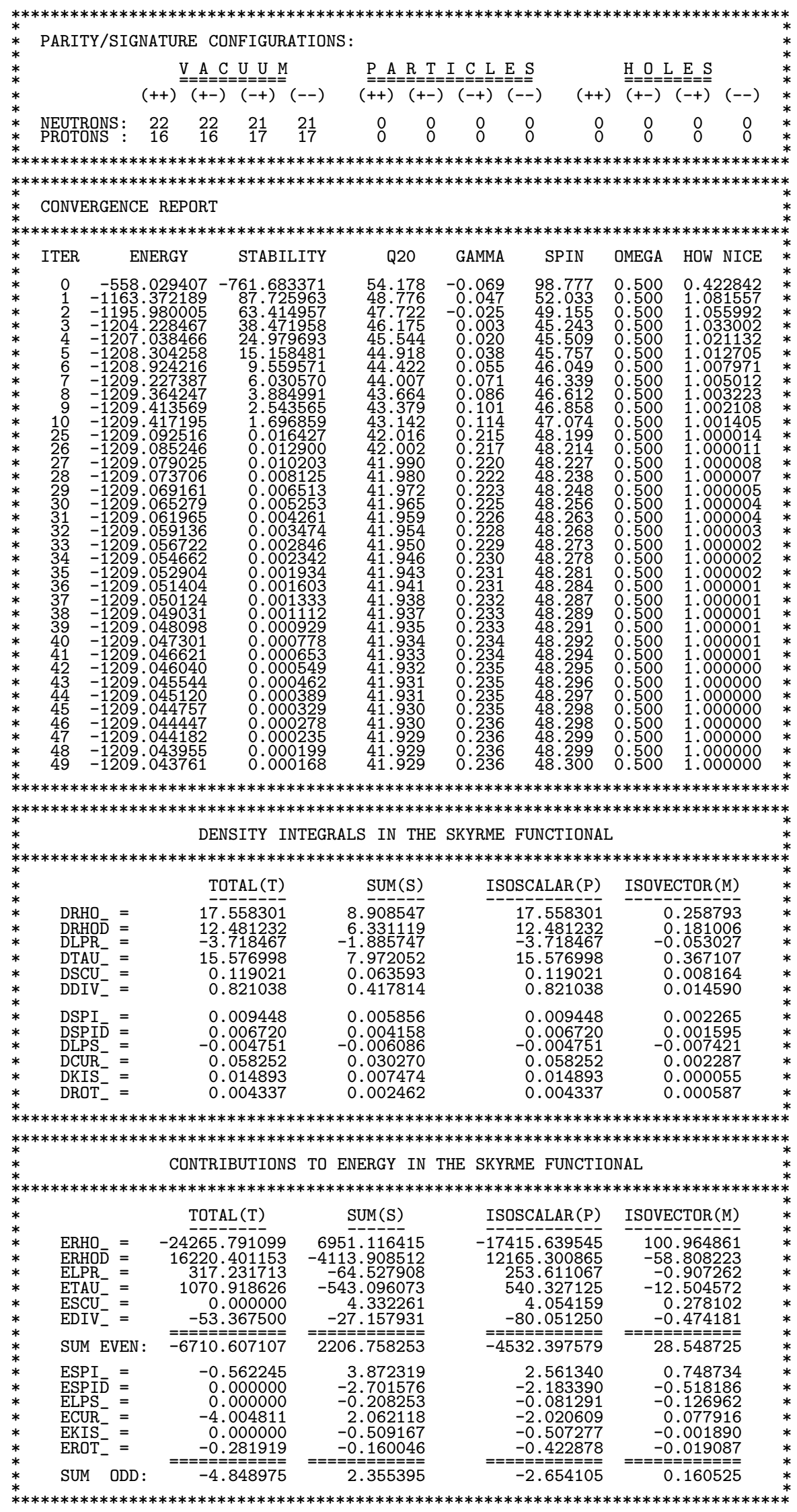




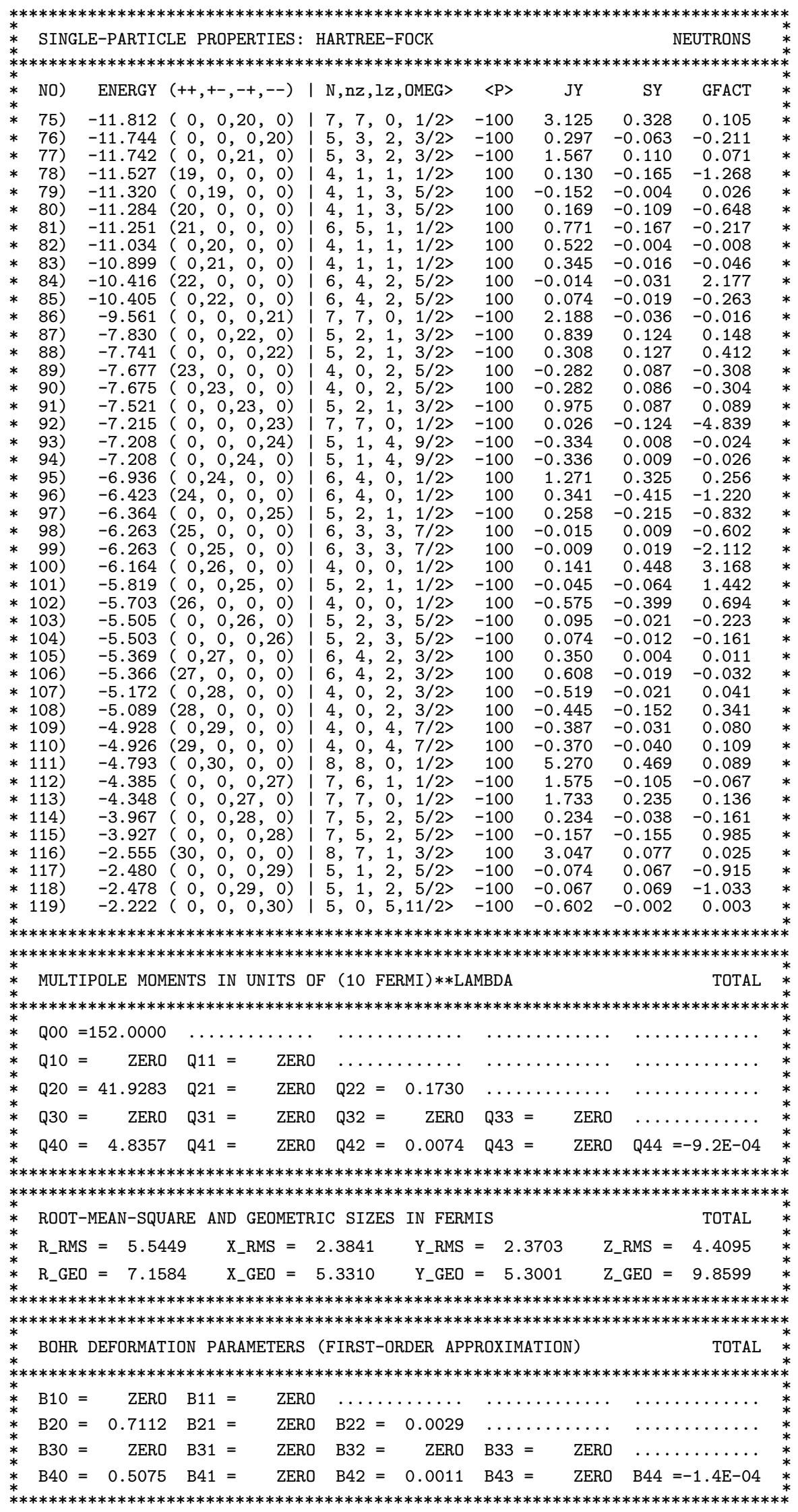




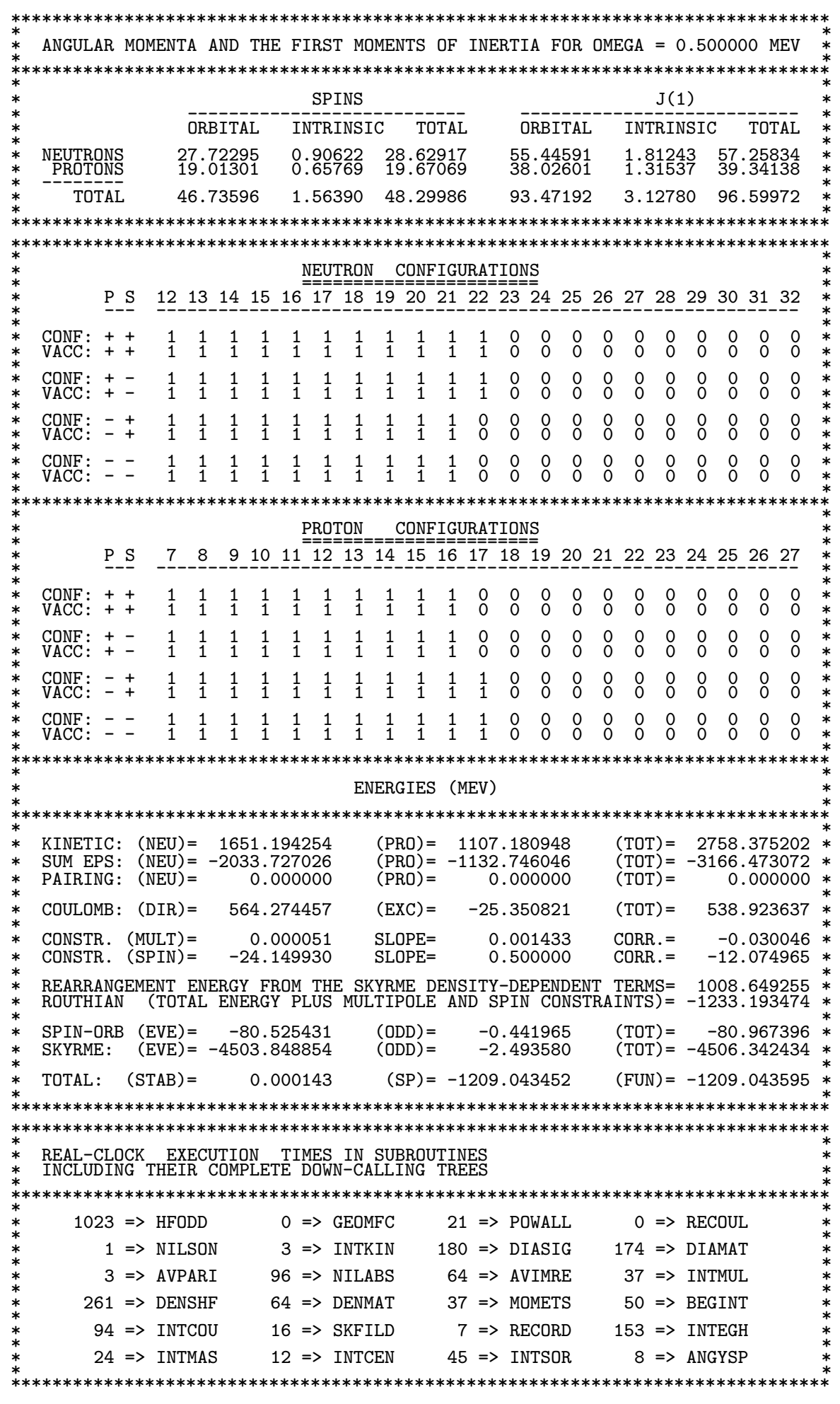

